\title{
The Gated Electrostatic Mass Spectrometer (GEMS): Definition and Preliminary Results
}

\author{
Federico A. Herrero, Hollis H. Jones, and Jeffrey G. Lee \\ Detector Systems Branch, Code 553, NASA Goddard Space Flight Center, Greenbelt, Maryland, USA
}

GEMS is a new type of time-of-flight mass spectrometer based on an electrostatic energy analyzer. Mass resolution equals the energy analyzer kinetic energy resolution, which is set by its slit size. In GEMS, monochromatic ions enter the entrance slit at random times, and the gated ion deflection produced by the electrostatic field in the analyzer rejects ions that are inside the analyzer at gate onset, detecting those entering the analyzer after gate onset. This provides mass separation while overcoming the temporal and spatial spread problems typical of TOF applications. Paradoxically, GEMS works because all ion masses follow identical trajectories. GEMS is easily multiplied into two-dimensional arrays to increase sensitivity in space applications, requires relatively low voltages, and uses only a few electrical connections. Thus, it is easy to package GEMS as a small, low-power instrument for applications in harsh environments. A disadvantage of GEMS is that its output is the integral of the TOF spectrum and the derivative of the raw data must be taken, a procedure that is likely to add noise. A version of GEMS detecting un-deflected ions ( $u$-GEMS) has been tested to demonstrate the time-integrated feature of the raw data but without the benefit of energy analysis. This paper describes GEMS implemented with the small deflection energy analyzer (SDEA), a compact version of the parallel plate energy analyzer. SDEA is described both analytically and with ion trajectory simulations using the ion trajectory simulation software SIMION; the results are then used to describe GEMS and compute its performance. (J Am Soc Mass Spectrom 2008, 19, 1384-1394) (C 2008 Published by Elsevier Inc. on behalf of American Society for Mass Spectrometry

$\mathrm{T}$ This section provides a brief review of space applications of mass spectrometers and the motivation toward miniaturization for ion and neutral composition measurements in space. Miniaturization provided the impetus for GEMS. Then there follows a discussion of mass spectrometers to recount (1) the role of magnetic-electric field sectors using continuous sources of ionization to achieve resolving power $M / \Delta M$ exceeding $10^{4},(2)$ the introduction of the time-of-flight (TOF) spectrometer using pulsed ion sources, and finally (3) the return to continuous sources, but now with TOF spectrometers. It is hoped that this may set the stage for the GEMS idea among other approaches to TOF spectrometry. Since GEMS is based on electrostatic energy analyzers (ESAs), a detailed description of the small deflection energy analyzer (SDEA) is given in the next section.

The earth's upper atmosphere and the atmospheres of other planets have been investigated with different types of mass spectrometers for more than five decades. Space applications have always required instruments of small volume, small mass, and low power. Current requirements for multipoint measurements in space

Address reprint requests to Dr. F. A. Herrero, Detector Systems Branch, Code 553, NASA Goddard Space Flight Center, Greenbelt, MD 20771, USA. E-mail: Fred.Herrero@nasa.gov pose an urgent need for further miniaturization over present levels. Several decades ago, Nier and Hayden [1] took advantage of the compactness of the MattauchHerzog geometry to fly a double-focusing mass spectrometer with a mass of about $4 \mathrm{~kg}$ and consuming less than $5 \mathrm{~W}$ of power. A similar instrument was later used to measure the composition of the Martian atmosphere [2]. Quadrupole and monopole spectrometers have been used in rocket flights [3] and in satellites [4], to investigate ion composition from $64 \mathrm{~km}$ and up [5] and the composition of the neutral air in the thermosphere [6]. The quadrupole mass filter was used in all of NASA's Atmosphere Explorer satellites and later in the Dynamics Explorer [6] and references therein. In those missions, the mass spectrometer provided not only composition, but was also used to sense the angular distribution of the atomic and molecular flux as it entered the spectrometer. Those data were used effectively to infer the perpendicular component (to the satellite velocity) of the wind and the air temperature at altitudes above $200 \mathrm{~km}$. The mass and power of these instruments ranged from 5 to $15 \mathrm{~kg}$ and 7 to $15 \mathrm{~W}$. As the collected data were used to further our understanding of the dynamics of the upper atmosphere, new scientific questions arose regarding small-scale features of the thermosphere and ionosphere, on their origins, and their evolution. These new questions require simul- 
taneous multipoint measurements that must be obtained in constellations of many (10 to 100) nanosatellites. The power buses of these small satellites provide no more than $4 \mathrm{~W}$ for all satellite functions, leaving at most $2 \mathrm{Ws}$ for the scientific instruments. The instrument mass is equally reduced to less than $2 \mathrm{~kg}$.

In a joint effort between the NASA Goddard Space Flight Center and the Naval Research Laboratory, a new type of energy analyzer has been developed and is being used to build a wind and temperature spectrometer (WTS) with total mass less than $1 \mathrm{~kg}$ and consuming less than $1 \mathrm{~W}$ of power $[7,8]$. The WTS sensor alone has a mass less than $0.1 \mathrm{~kg}$ including the detector, the electronics occupying the rest. However, the required power is well within the $1 \mathrm{~W}$ envelope. Another version of WTS has been designed for the University of Colorado to launch in 2010 [9], with mass/power less than $1 \mathrm{~kg} / 1 \mathrm{~W}$. Both versions of WTS use the SDEA described below. It is worth noting that WTS behaves in low-Earth-orbit as a very coarse mass spectrometer, determining mass from energy spectra with resolving power of about three [8].

Mass spectrometers use an ion source to convert the neutral sample (gas, liquid, or solid) into an ion beam for analysis in an ion mass spectrometer; that is, a charged-particle spectrometer that separates masses according to momentum, velocity, gyro-frequency, or some other convenient property. In momentum or velocity analyzers, the energy spread of the ion beam was a problem, limiting mass resolution until the 1930s when the elegant technique of double focusing was developed to deal with that problem by combining electric field sectors with magnetic field sectors [10]. The magnetic mass sectors operated with ion sources that ran continuously, and the ions could be dispersed to achieve resolving powers about $10^{5}$ and higher. These instruments had size scales about $1 \mathrm{~m}$ and the slits used were about $0.020 \mathrm{~mm}$ to achieve the desired resolving powers [11]. They were very large, with little portability, and often the magnetic fields interfered with the operation of other devices nearby.

The TOF mass spectrometer offered a way to eliminate the large magnetic sectors and perhaps lead to instruments of reduced size (see Mirsaleh-Kohan et al. [12] for a comprehensive review). Stephen [13] introduced the TOF idea, and Cameron and Eggers [14] first demonstrated it. The basic concept of TOF is that an ion pulse moving at fixed energy will spread along a drift length until different masses are separated in time sufficiently to be detected separately. In general, long drift lengths improve resolving power. Early (nonmagnetic) TOF instruments had poor resolution due to ion source energy spread. Wiley and McLaren [15], noting that part of the energy spread was due to the spatial variation of the electrostatic potential in the ion source, developed the space-focusing idea. Since the most energetic ions came from the back of the ion source, they could catch up with the less energetic ones on their way to the detector. Therefore, there had to be a position along the ion beam where all ions would arrive at the same time if the energy spread was due to position spread. However, this position-time focus is massdependent for an ion beam of fixed energy and could only work over a narrow mass range. Refinements on the Wiley-McLaren [15] idea are reviewed in Guilhaus [16] and Mirsaleh-Kohan et al. [12]; perhaps the most elegant technique to deal with the energy spread problem is the Reflectron technique - an implementation of the harmonic oscillator problem in TOF to bring all ions back to a space-time focus independently of their ion energy $[17,18]$. The Reflectron is an effective way to increase to drift length of the TOF spectrometer with little increase in the instrument size. These TOF applications relied on pulsed ion sources with inherent temporal and spatial spreads whose effect was minimized taking advantage of the energy spread.

More recently, continuous ion sources have begun to appear in TOF-MS [19], mainly in the orthogonal acceleration TOF-MS in which a continuous ion beam is subjected to pulsed acceleration in a direction perpendicular to its original axis. One distinct advantage of this technique is that the effect of the energy spread (along the ion beam axis) effect is reduced significantly in proportion to the angular divergence of the beam in the extraction region. In addition, good collimation to provide a very narrow ion beam at the point of orthogonal acceleration has been exploited to further enhance the resolving power. This technique, first reported by Dawson and Guilhaus [20], is capable of resolving powers of 3000 to 5000 (FWHM).

The GEMS idea presented here operates with a continuous ion source and its mass resolution is set strictly by the kinetic energy resolution of the electrostatic energy analyzer, and this is ultimately controlled by the slit size. In this sense, GEMS represents a return to the magnetic sector of the 1930s, but without a magnet, and with much smaller scale and other possibilities that require developments in other areas such as high quality ion beams. Slit sizes of 0.002 to $0.05 \mathrm{~mm}$ are feasible in our laboratory with micro-machining techniques. Using the equations derived below, a $0.010 \mathrm{~mm}$ slit width and an energy analyzer gap of $10.0 \mathrm{~mm}(1 \mathrm{~cm})$ provides a resolving power of 1000 .

\section{Resolution and Resolving Power}

GEMS brings together two different cultures in the area of charged-particle spectrometers with somewhat different definitions of the terms resolution and resolving power. According to de Hoffman and Stroobant [21], "The exact definition of these terms is one of the more confusing subjects of mass spectrometry terminology that continues to be debated." Strictly speaking, in a charged-particle spectrometer, be it an energy analyzer or a mass spectrometer, the term resolution refers to the width of the peak in energy or mass. Indeed, it is the width that determines the narrowest spacing between two peaks that are separated or resolved as two; the 
narrower the width, the higher the resolving power. In mass spectrometers and energy analyzers, the width $\Delta M$ of the mass peak or $\Delta K$ of the energy peak generally increases in direct proportion to the mass or energy. Thus, it is most convenient to use the ratios $\Delta M / M$ and $\Delta K / K$, for they are usually simple functions of the instrument geometry [22, 23]. In addition, when specifying overall peak shape due, for example, to spreads or widths in spatial, temporal, and other sources of energy spreads, it is the sum in the squares of the widths that arises in a natural way because of their independent probabilities of occurrence [16]. This article requires the use of terms like $\Delta K / K$ in the discussion of the energy analyzer. Therefore, the term resolution is used for $\Delta K / K$ or $\Delta M / M$. The more descriptive term $M / \Delta M$ (or $K / \Delta K$ ) will be called resolving power, as used by Guilhaus [16].

The article begins with a description of the smalldeflection energy analyzer (SDEA) since this is the physical basis for GEMS. We then proceed to a description of the operation of GEMS, show preliminary results obtained with $u$-GEMS (the undeflected ion version of GEMS), and discuss likely applications of this new approach enabled by the characteristics of the device.

\section{The Small Deflection Energy Analyzer (SDEA)}

Figure 1 shows the basic geometry of a small-deflection energy analyzer (SDEA). The angle $\alpha$ shown is exaggerated for the sake of illustration. On the left panel (side

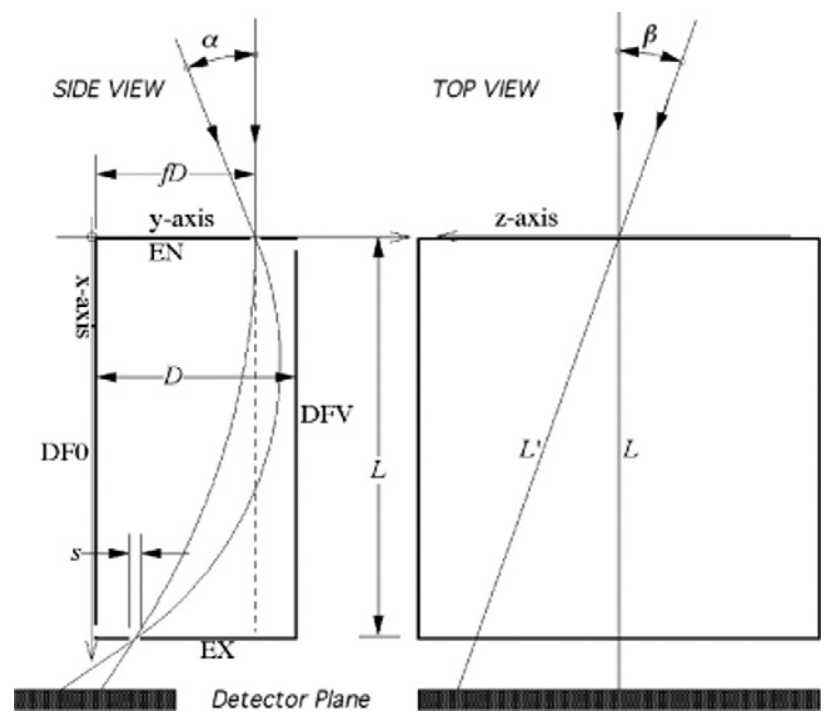

Figure 1. The side view of a small deflection energy analyzer (SDEA) on the left shows two ion trajectories incident from above one perpendicular to the entrance aperture plane EN. The elevation angle $\alpha$ is shown. The top view on the right shows the same two trajectories, again on perpendicular to the entrance plane and the other incident with lateral angle $\beta$. The ions pass the exit plane EX through the slit with width sequal to that of the entrance slit. Planes DF0 and DFV are the deflector plates and are each biased at 0 and $\mathrm{V}$ volts, respectively. Additional details are given in the text. view) are shown the entrance plate $\mathrm{EN}$ tied to the deflector plate DF0, and the exit plate EX tied to deflector plate DFV. Plates EN and EX contain the entrance and exit aperture slits, respectively, both with the same width $s$. $L$ is the length of the deflector plates DF0 and DFV, and $D$ is the gap between them. $L$ runs parallel to the $x$-axis and $D$ parallel to the $y$-axis. Plates EN-DF0 are biased at $0 \mathrm{~V}$ with respect to the ionization region and plates EX-DFV at $V$ volts DC. The electric field formed in the gap is distorted by EN and EX. However, SIMION trajectory simulations show that trajectories obtained are essentially parabolic when $L>4 D$; thus, for the analytical trajectory calculations carried out here, it is reasonable to assume a uniform field in the energy analyzer gap.

The left panel of Figure 1 (side view) shows two ion trajectories that pass the centers of both the entrance and exit slits, one with angle of normal incidence $\alpha=0^{\circ}$ and the other with $\alpha \neq 0$. These trajectories are called central trajectories because they pass through the centers of both slits. Figure 1 also shows that the central trajectory has initial $y$ position at $y_{i}=f D$ where $0.8<f<0.95$ in order to achieve significant ion deflection inside SDEA.

The right panel shows the top view of two ion trajectories one with incident angle $\beta=0$ and one with $\beta \neq$ in the $x-z$ plane. The length of the trajectory in the $\mathrm{x}-\mathrm{z}$ plane is $L^{\prime}(\beta)=L / \cos \beta$, and in this plane it is a straight line, since there are no fields along the direction of the $\mathrm{z}$-axis. The field near the edges of the plates (top view on right panel) may be rendered uniform attaching resistive glass plates electrically to plates DFV and DF0 and provide continuous variation of the potential at the left and right edges to impose the potential distribution that corresponds to a uniform field [24]. Using this new technology allows reduction in the lateral dimension of GEMS.

With the above provisions, consider the three components of the electric field as follows: $\varepsilon_{x}=0$, $\varepsilon_{y}=V / D, \varepsilon_{z}=0$ with equations of motion $\ddot{x} Y Y=0$, $\ddot{y} Y Y=-(q / m) V / D, \ddot{z} Y Y=0$ where $q=Z e$ is the total charge on the ion with $Z=1,2,3, \ldots$, and $e$ the electronic charge. An ion with kinetic energy $K=$ $m v^{2} / 2$, incident with direction angles $(\alpha, \beta)$, will describe a parabolic trajectory in the $x-y$ plane and a straight-line trajectory in the $\mathrm{x}-\mathrm{z}$ plane, as shown in Figure 1. Solving the equations of motion for such an ion entering SDEA at angles $(\alpha, \beta)$ at $x_{i}=0, y_{i}=f D$, $z_{\mathrm{i}}=0$ and exiting at $\left(x_{f}=L, y_{f}=(1-f) D, z_{f}=L \tan \beta\right)$, the net displacement of the ion in the y-direction is $y_{c}=$ $(2 f-1) D$. The transmission condition that must be satisfied by the kinetic energy for an ion to pass the exit slit is

$$
Y_{C}=\frac{Z}{4} \frac{V}{K} \frac{L^{\prime}(\beta)^{2}}{D \cos ^{2} \alpha}-L^{\prime}(\beta) \tan \alpha
$$

In eq 1, the kinetic energy is expressed in $\mathrm{eV}$ and the voltage $V$ in volts. 
Equation 1 may be rearranged to give the transmitted ion kinetic energy $k$ for any given voltage $V$ and angles $(\alpha, \beta)$ :

$$
K=\frac{Z V}{4}\left(\frac{L^{\prime}(\beta)}{D}\right)^{2} \frac{D}{y_{C} \cos ^{2} \alpha+L^{\prime}(\beta) \sin \alpha \cos \alpha},
$$

The energy resolution $R=\Delta K / K$ of the SDEA may now be obtained with the aid of eq $2 ; \Delta K / K$ is usually given as a fraction or a percentage. $\Delta K$ is the passband; that is, the range of energies that the spectrometer transmits at fixed volage $V$. All variables controlling the transmitted energy (i.e., $s, \alpha, \beta, V$ ) are independent; therefore, the net energy spread will be the result of the sum in quadrature of all the allowed ranges in all the variables that determine the transmitted energy $[16,25]$. The net energy spread may then be written:

$$
(\Delta K)^{2}=\left(\Delta K_{s}\right)^{2}+\left(\frac{\partial K}{\partial \alpha} \Delta \alpha\right)^{2}+\left(\Delta K_{\beta}\right)^{2}+\left(\frac{\partial K}{\partial V} \Delta V\right)^{2}
$$

The first term on the right, $\Delta K_{s}$, corresponds to the allowed energy passband due to the slit width $s$, which is an implicit variable in the function $K$ of eq 2 , but will appear explicitly in the derivation below. The second and fourth terms represent the allowed ranges in angle $\alpha$ and voltage $V$ in terms of the corresponding partial derivatives because the function $K$ is an odd function $\alpha$ and $V$. The third term must be calculated differently because $K$ is an even function of $\beta$ and its first derivative vanishes. In other cases there may be additional parameters that affect the passband. In this case, there are only the four variables $(s, \alpha, \beta, V)$. The following paragraphs show how to evaluate the four terms that determine the energy passband. The approach with eq 3 is to determine the first term based on the criterion of full-width at half-maximum for the desired passband. Then the ranges $\Delta \alpha$ and $\Delta \beta$, and $\Delta V$ are considered. A sound design approach is to set these ranges such that none of the terms exceed the first. Thus, the net energy passband of the spectrometer may be specified to be equal to or less than $\sqrt{N \Delta K_{s}}$, where $N$ is the number of independent variables; $N=4$ in this case.

Dividing eq 3 by the square of the mean transmitted energy $K$ and taking the square root gives an expression for the energy resolution:

$$
\begin{aligned}
\left(\frac{\Delta K}{K}\right)_{F W H M}= & \left(\left(\frac{\Delta K_{s}}{K}\right)^{2}+\left(\frac{\Delta K_{\alpha}}{K}\right)^{2}\right. \\
& \left.+\left(\frac{\Delta K_{\beta}}{K}\right)^{2}+\left(\frac{\Delta K_{V}}{K}\right)^{2}\right)^{1 / 2} .
\end{aligned}
$$

To determine $\Delta K_{s} / K$, consider a bundle of rays (trajectories), all with the same kinetic energy and with normal incidence $\left(\alpha=0^{\circ}, \beta=0^{\circ}\right)$. The rays are evenly spaced and in sufficient number to fill the entrance slit uniformly. Then, when the energy is such that the central ray passes the exit slit at its very center, all rays in the bundle pass the exit slit and transmission is 100\%. This energy corresponds to the peak of the transmission passband and is given by eq 2 . As the energy changes, it follows that when the central ray in the bundle grazes one of the edges of the exit slit, only half the rays pass the exit slit and transmission is half-maximum. For any given applied voltage $V$ this can happen only at two energies, and those two energies represent the edges of the transmission passband for the half -maximum transmission. For the highest energy $K_{h}$, the ray deflects least and thus grazes the right edge of the slit; for the lowest energy $K_{l}$, the ray deflects the most and grazes the left edge of the exit slit. This notion determines the full width at half-maximum, as shown in the following paragraph.

Since $K_{h}$ is deflected least, the $y_{C}$ term in the denominator of eq 2 is replaced by $y_{C}-s / 2$. Correspondingly, since $K_{l}$ is deflected the most, the term $y_{C}$ is replaced by $y_{C}+s / 2$. This gives the energy difference $\Delta K=K_{h}-K_{l}$ which, when divided by the mean energy $K$ (given by eq 2 exactly) gives

$$
\left(\frac{\Delta K_{s}}{K}\right)=\frac{s}{\left(y_{C}^{2}-s^{2} / 4\right)} y_{C}
$$

In SDEA applications, desired energy resolutions are such that $R<20 \%$. This means that $s<0.2 y_{c}$. Therefore the $s^{2} / 4$ term in the denominator of eq 4 may be ignored, and the energy resolution is well represented by

$$
\left(\frac{\Delta K_{s}}{K}\right)=\frac{s}{y_{C}}=\frac{s}{(2 f-1) D}
$$

For applications requiring resolutions better than $5 \%$ $(R<0.05)$, the factor $f$ should be between 0.8 and 0.95 . In this case, reasonable energy resolution estimates are given by the ratio $s / D$; that is, $R=\Delta K / K \approx s / D$, a useful result to apply at the beginning of the design process.

The variation of $K$ with angle $\alpha$ follows from the partial derivative of $K$ with respect to $\alpha$ evaluated at $\alpha=0$, and then divided by $K$; that is,

$$
\left(\frac{\Delta K_{\alpha}}{K}\right)=\frac{1}{K}\left(\frac{\partial K}{\partial \alpha}\right)_{\alpha=0} \Delta \alpha=\frac{L}{(2 f-1) D} \Delta \alpha .
$$

The range $\Delta \alpha$ of the angle of incidence can be determined from the design requirements. For example, a likely value for $L / D$ is, say 4 , and with $f=0.9, \Delta K_{\alpha} / K$ is $5 \Delta \alpha$. If the energy resolution is, say, $5 \%$, then $\Delta \alpha$ must be less than about $0.01 \mathrm{rad}$ (about $0.6^{\circ}$ ).

The effect of the field of view in the angle $\beta$ may be obtained also from eq 2. Recognizing that the angle $\alpha$ is less than $1^{\circ}$ simplifies eq 2 to the form $K=A / \cos ^{2} \beta$ where $A=Z V L^{2} /\left(4 y_{c} D\right)$ is a constant. This expression is an even function of $\beta$. Therefore its first derivative will 


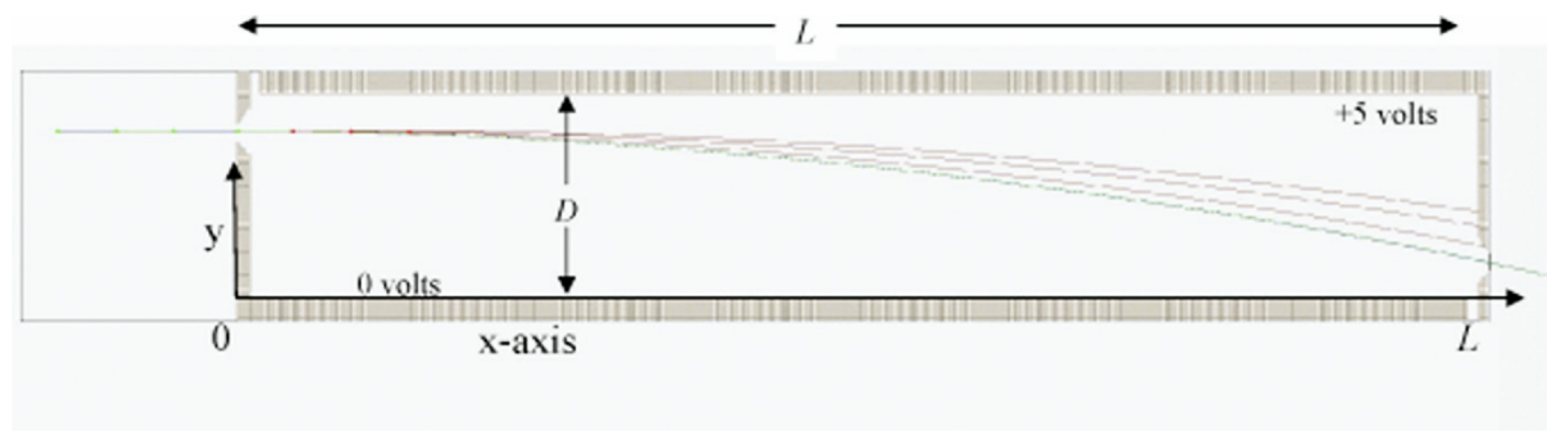

Figure 2. How GEMS works: SIMION trajectories of seven ions moving horizontally from the left. All ions have the same kinetic energy, about $43 \mathrm{eV}$. The deflection voltage $(+5 \mathrm{~V})$ is gated on at time $t=0$; ion positions at $t=0$ shown by seven dots. The four leftmost dots (ions) undergo full deflection and pass the exit slit at right. The three internal dots (ions) do not sufficient space to develop full deflection and do not exit.

not contribute to the energy spread sum in eq 3 , though $\beta$ will have an effect on the energy passband. In this case, the energy spread is $\Delta K_{\beta}=K(\beta)-K(0)=$ $A / \cos ^{2} \beta-A$. Then it follows that

$$
\left(\frac{\Delta K_{\beta}}{K}\right)=\sin ^{2} \beta
$$

The largest allowable value of $\beta$ that is consistent with an energy resolution of $5 \%$ must then be $\beta_{\text {mas }}=\sin ^{-1}$ $(\sqrt{0.05})=12.9^{\circ}$ Since the transmitted energy is an even function of $\beta$, the extent of the field of view in $\beta$ is $\Delta \beta=2 \beta_{\max } \cong 26^{\circ}$. The large value of $\Delta \beta$ in comparison with $\Delta \alpha$ is a characteristic of the SDEA that may be used to advantage in those cases where sensitivity is an issue and angular coverage is required (e.g., in an imaging mass spectrometer application).

For the case of GEMS using a small circular (or square) aperture, the exit slit may be laid out along a circular arc in the $\mathrm{x}-\mathrm{z}$ plane of Figure 1 so that all trajectories have the same length $L$ along the SDEA independent of angle $\beta$. In that case, the resolution term due to $\beta$ is determined by the $\Delta \beta$ subtended by a detector pixel beyond the exit slit. This opens up the possibility of imaging the composition of the gas incident on an "imaging mass spectrometer" from, e.g., gaseous plumes emanating from the surface of the object under study. Such imaging applications of composition may be required in asteroid, comet, and lunar surface investigations soon.

The last term in eq 4 follows immediately from eq 2 since the transmitted kinetic energy is directly proportional to the applied deflector voltage. This term is

$$
\left(\frac{\Delta K_{V}}{K}\right)=\frac{\Delta V}{V}
$$

Voltage supply ripple and line noise in all parts of the spectrometer from the ion source to the analyzer can contribute. Voltage supply ripple of $1 / 4000$ is typical and with some care this can be reduced by a factor of 10 or more. But it is important to note that it could be a limiting factor in the ultimate mass resolution achieved with GEMS.

\section{The Gated Electrostatic Mass Spectrometer (GEMS)}

It is now possible to describe the operation of the SDEA as a GEMS mass spectrometer. Consider the SDEA cavity in Figure 1 again. If the deflection voltage on DFV is 0 initially, all incident ions that pass the entrance slit simply continue along straight-line trajectories as shown by the dashed line on the side view. If the voltage is gated at time $t=0$, ions that were already inside the cavity will begin to deflect but not sufficiently to pass the exit slit because they do not have sufficient distance along the $\mathrm{x}$-axis before reaching the back plate. Ions that were outside the cavity at $t=0$ will proceed to traverse the entire cavity and thereby undergo a full deflection and pass the exit slit.

Figure 2 portrays this idea with actual SIMION trajectory simulations. It shows a SDEA cavity turned on, so its $x$-axis is now along the horizontal. This illustrates the GEMS operation with several ion trajectories that originate at different points along the undeflected (horizontal) path of the incident ions. The seven small dots along the trajectories show the positions of the ions at the time the gate voltage is turned on, that is, at $t=0$ the top plate voltage goes from 0 to $+5 \mathrm{~V}$ ). Three of the dots are inside the SDEA cavity, thee outside, and one at the entrance slit. The three trajectories striking the back plate correspond to the three dots that are inside the SDEA cavity. The other four trajectories managed to pass the slit as shown. So, paradoxically, the realization of a gated mass spectrometer with SDEA hinges on the fact that the ion trajectories are independent of mass.

However, note that depending on the size of the slits, ions that are inside the cavity but sufficiently close to 
the entrance may undergo enough deflection to pass the exit slit. The question of how close ions inside SDEA can be to the exit slit and still pass is examined now in detail to yield an answer in the form of a relation between the energy resolution of SDEA and the mass resolution of GEMS.

Clearly, ions that pass the exit slit are either at the slit $(x=0)$ or outside the cavity $(x<0)$. Now for $x>0$, one asks how close to the slit the ions can be and still pass the exit slit. All the trajectories shown in Figure 2 are parabolic to a very good approximation and they all have a negative slope in the $x-y$ plane. Therefore, when a trajectory that begins at the entrance plane $(x=0)$ is displaced to the right by $\Delta x$, its intercept with the exit plane moves upward an amount $\Delta y$ that follows from eq 1 setting $\alpha=\beta=0$ :

$$
\Delta y=\left(\frac{\partial y_{C}}{\partial L}\right) \Delta x=\frac{Z V L}{2 K D} \Delta x .
$$

Now consider a central trajectory starting at $(x=0, y=$ $f D, z=0$ ) passing with $\alpha=0$ through the center of the entrance slit and with energy $K_{h}$ as in the discussion preceding eq 5 . This trajectory grazes the upper edge of the exit slit allowing half the rays in its bundle to exit. As the origin of the trajectory shifts to the right even a small amount, its passage is blocked since its intercept with the exit plane $(x=L)$ moves upward. The same trajectory, but with the lower energy $K_{l}$ used for eq 5 , will graze the lower edge of the exit slit if it starts at $(x=0, y=f D, z=0)$ as its origin shifts to the right an amount $\Delta x$ such that $\Delta y=s$ in eq 10. The fraction of rays in the bundle will change from 0.5 to 1 and back down to 0.5 for such a $\Delta x$ translation. Setting $\Delta Y=s$ in eq 10 and using the fact that the time to traverse the SDEA cavity is $t=L / v_{x}$ and $\Delta t=\Delta x / v_{x}$ gives that

$$
\frac{\Delta t}{t}=\frac{1}{2} \frac{s}{D} \cong \frac{1}{2} \frac{\Delta K}{K}
$$

The time for an ion of mass $m$ to traverse the distance $L$ is proportional to $(m)^{1 / 2}$, and therefore $\Delta t / t=$ $1 / 2 \Delta m / m$; thus, the resolving power $P$ of GEMS, being equal to the inverse of its energy resolution $(P=1 / R)$, is

$$
P=\frac{m}{\Delta m}=\frac{K}{\Delta K} \cong \frac{D}{s} .
$$

In summary, GEMS operates with monochromatic ions incident on the analyzer entrance slit at random times. It "opens" its entrance gate at start time $(t=0)$ and detects all ions passing the entrance thereafter because they deflect fully in the electric field "opened" by the gate. Ions that are inside the analyzer at $t=0$ do not have sufficient distance to deflect far enough to clear the exit slit and reach the detector. Thus, for the $i$ th mass, the earliest possible arrival time $t_{a i}$ at the detector corresponds to the ion being at the entrance at $t=0$; the lightest mass has the smallest $t_{a i}$. Though ions are randomly spaced, they reach the detector at an average uniform rate, but only after time $t_{a i}$ for each ion mass $i$. As a result, after a large number of ions have been detected (i.e., $10^{5}$ ), the accumulated ion arrival times give a distribution in the number of arrivals versus arrival time in the form of step functions added together, one step for each $t_{a i}$ (ith mass).

\section{Simulated Mass Spectra and Expected Performance}

Figure 3 shows simulated GEMS spectra to illustrate the nature of the expected data. The simulation used $25 \mathrm{eV}$ for the kinetic energy, a length $L=1.0 \mathrm{~cm}$, a gap $D=$ $0.18 \mathrm{~cm}$ and a slit width $s=0.05 \mathrm{~mm}$. With these
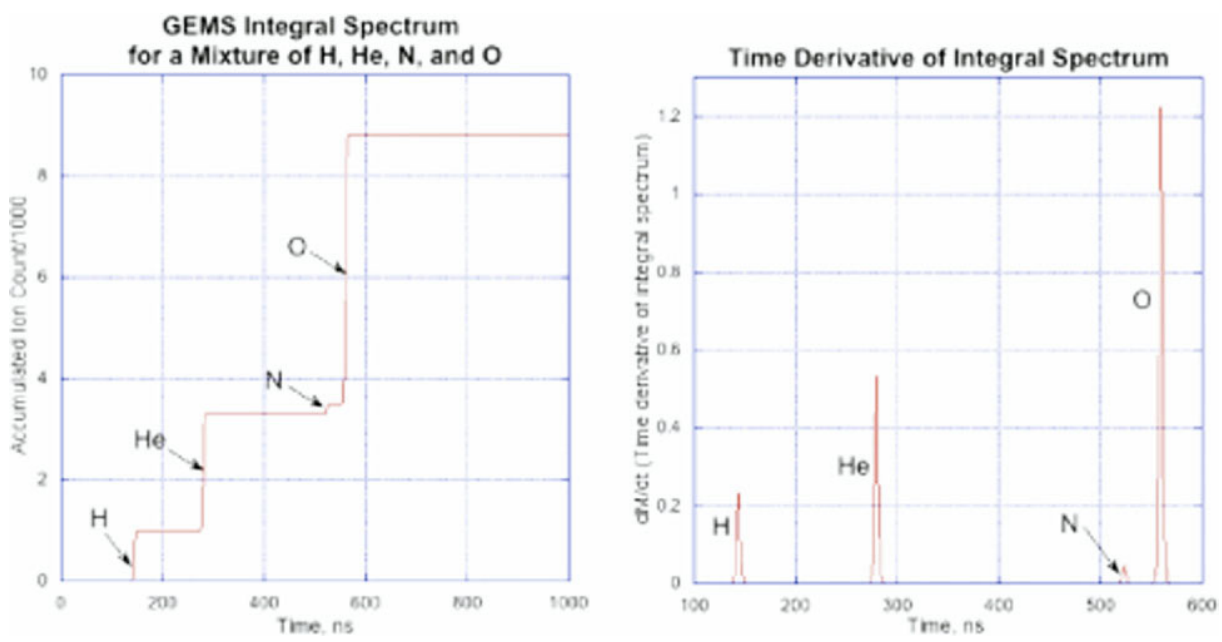

Figure 3. Predicted performance of GEMS. The left panel shows a simulated spectrum of an ion beam containing singly charged $\mathrm{H}, \mathrm{He}, \mathrm{N}$, and $\mathrm{O}$ ions. The simulated kinetic energy was $25 \mathrm{eV}$, and SDEA had length $L=10.0 \mathrm{~mm}$, gap $D=1.8 \mathrm{~mm}$, and slit width $s=0.05 \mathrm{~mm}$. 

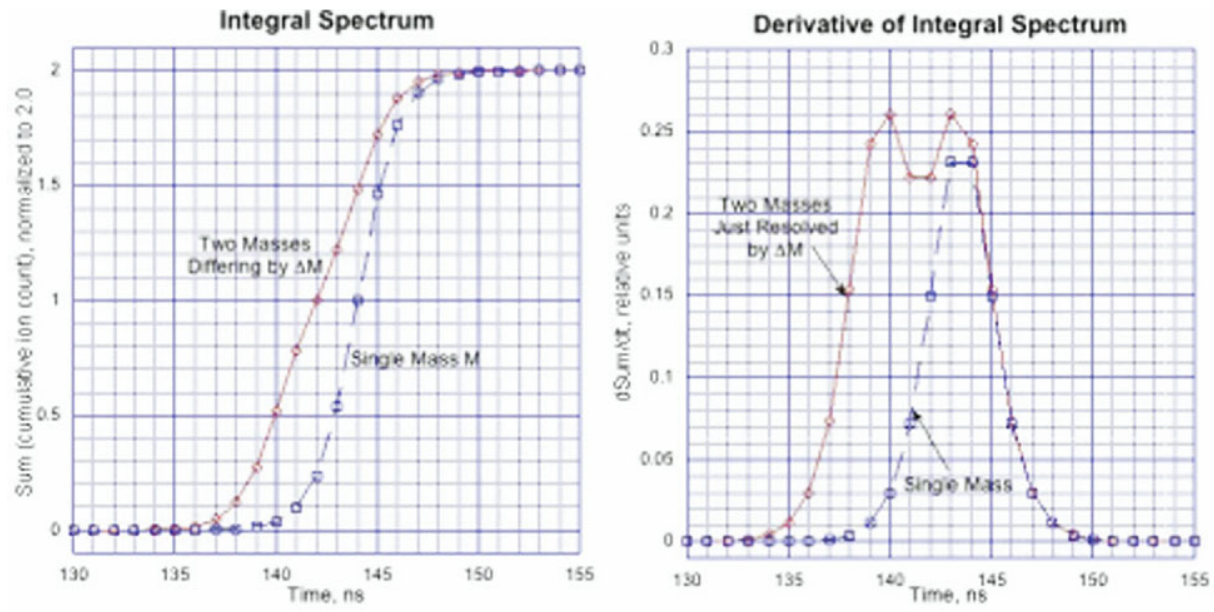

Figure 4. Shape of the integral spectrum with two masses of equal ion beam abundance, and differing only by the limit of resolution.

numbers, the simulated resolving power $m / \Delta \mathrm{m}$ is about 18. The composition of the ion beam contains singly charged ions of $\mathrm{H}, \mathrm{He}, \mathrm{N}$, and $\mathrm{O}$. The left panel shows the $\mathrm{H}$ ions with the earliest arrival time of about $140 \mathrm{~ns}$ followed by He ions at about $280 \mathrm{~ns}$. The least abundant in this example is atomic nitrogen $\mathrm{N}$ whose ions show an arrival time of about $524 \mathrm{~ns}$ and then the most abundant atomic oxygen $\mathrm{O}$ ions arrive at $560 \mathrm{~ns}$. The spectrum is clearly the sum of step functions, one for each mass, and as they add up, they build up the shoulders shown. So, the observations represent a cumulative or integral spectrum. Taking the derivative of the integral spectrum then gives the normal TOF spectrum shown on the right.

One of the disadvantages of GEMS is that it delivers the integral of the mass spectrum instead of the spectrum itself. This may make it difficult to detect a minor constituent if its relative abundance in the ion beam is below a certain limit. At this point, the nature of the difficulty may be examined by looking at the shape of the integral spectrum curve. Figure 4 shows the integral spectrum for two masses that are very close together, close to the resolution limit, but of equal abundance. Instrument resolution in time is $\Delta t / t=1 / 36$. The shape of the shoulder in the integral mass spectrum must be supported by sufficient cumulative ion counts to allow the derivative of the data. The panel on the left compares the shoulder due to a single mass with the shoulder developed by two masses separated only by $\Delta t=t / 36$. The panel on the right shows the derivative of the two plots to indicate the single mass peak and the two mass peaks just resolved. One could argue that anytime the shoulder slope is less than expected based on $\Delta t / t$ one should suspect multiple masses. Figure 4 shows that under conditions of good signal levels, it may be possible to detect the variation in slope associated with the double mass peak. Of course the situation is far more complicated when the two abundances differ, but the figure does show that even with less than optimum signal the shallower slope observed may be taken as indication that there are two masses represented in the shoulder. This issue clearly deserves further study, taking into account noise and stability and comparing performance with high-resolution TOF-MS such as one that uses both orthogonal extraction and a reflectron [19].

\section{Preliminary Test: $u$-GEMS}

It proved convenient to demonstrate the basic idea of GEMS in a version of it that may be called $u$-GEMS, for it analyzes undeflected ions in a drift length directly in line with the detector.

The experimental configuration was easily adopted from that used to test instruments for space flight, and it is described with reference to Figure 5 showing the experimental setup for $u$-GEMS. Ions extracted from the ion source I.S. at A on the left are focused at aperture C. The collimated ion beam passes aperture $C$ and drifts toward the detector at $\mathrm{D}$ in the field-free regions between $B$ and $C$, and $C$ and $D$ at the kinetic energy set before reaching the deflector $\mathrm{B}$. The ion source $(\mathrm{Co}-$ lutron Research Corporation, Boulder, CO) operated at relatively high-pressure (about $10^{-2}$ Torr) with an ex-

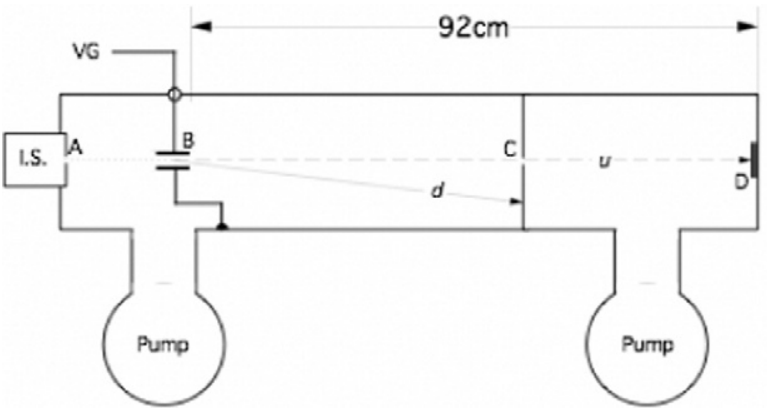

Figure 5. Experimental setup for $u$-GEMS. 
tractor lens (not shown) at the aperture A biased at $-1000 \mathrm{~V}$ with respect to A. An Einzel lens (not shown) served to focus the ion beam at aperture $C$ whose diameter was $1 \mathrm{~mm}$ to provide significant differential pumping between the ion source chamber (A to $C$ ) and the detector chamber ( $C$ to $D$ ) on the right. Ion energy was set by the voltage of the ion source anode with respect to ground. The chamber on the left was pumped by a turbo-molecular pump with a pumping speed of $400 \mathrm{l} / \mathrm{s}$; the detector chamber was pumped by a $1200 \mathrm{l} / \mathrm{s}$ cryopump. The approximate respective pressures were $2 \times 10^{-5}$ Torr and $1 \times 10^{-8}$ Torr, respectively. The ion source and detector chambers were purged with dry nitrogen gas overnight before the experiment. Pure $\mathrm{N}_{2}$ gas in the ion source discharge is expected to give a relatively simple spectrum in the ion beam, having predominantly $\mathrm{N}^{+}$and $\mathrm{N}_{2}^{+}$to facilitate the demonstration. $200 \mathrm{eV}$ ions were focused and collimated through the $1 \mathrm{~mm}$ aperture, traveling a distance of about $92 \mathrm{~cm}$ at constant kinetic energy between the deflector $B$ and the detector at $D$. The deflection gate voltage VG was activated by a square wave generator switching from $0 \mathrm{~V}$ to $10 \mathrm{~V}$ with a period $T=60$ microseconds (50\% duty cycle). The detector used was a $25 \mathrm{~cm}$ diameter microchannel plate chevron pair (MCP) with a gain of about $3 \times$ $10^{6}$. Ion pulses from the MCP were fed to a TOF electronics card, Quantar model P7889 (Quantar Technology Inc., Santa Cruz, CA). The P7889 measures the arrival of STOP input events as given by each ion arrival at D, relative to a previous START signal, given by the gate voltage transition from 10 to $0 \mathrm{~V}$ ). The measured data were transferred to a PC as they were acquired where the number of arrivals corresponding to every time channel was recorded.

Thus, at time $t=0$, the gate voltage VG goes from 10 to $0 \mathrm{~V}$ and remains at $0 \mathrm{~V}$ for half the period of the square wave $(T / 2=30 \mu \mathrm{s})$ to allow ions to pass the deflector and on to the detector, as shown by the trajectory labeled $u$. During the half-period that the voltage is at $+10 \mathrm{~V}$, the ions are deflected away from the aperture $C$, as shown by trajectory labeled $d$. The earliest time of arrival at the detector for an ion of mass $m_{i}$ corresponds to the ion being just to the right of deflector $B$ at $t=0$. That time is given by

$$
t_{a i}=72 \times 10^{-6} \mathrm{~L} \sqrt{m_{i} / K} \text { seconds, }
$$

with $L$ in $\mathrm{m}, m_{i}$ in Daltons, and kinetic energy $K$ in $\mathrm{eV}$. $L$ is the distance between the deflector and the detector, $\sim 0.92 \mathrm{~m}$. At times later than $t=t_{a i}$, ions between $\mathrm{A}$ and $\mathrm{B}$ and ions exiting the ion source arrive at the detector at random, and with large numbers of ions accumulated, the integral spectrum of Figure 3 should be observed. At $t=T / 2$ VG switches from 0 back to 10 and the space between B and D is depleted of fast moving ions; at $t=T$, the detection cycle begins again and continues with period $T$.

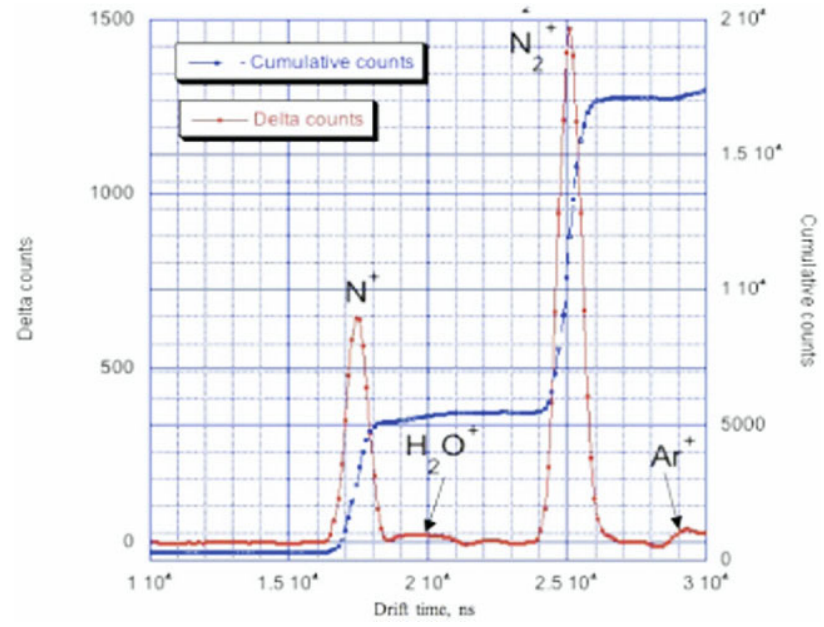

Figure 6. Mass spectrum obtained with $u$-GEMS using a $200 \mathrm{eV}$ ion beam using pure nitrogen gas in the ion source. Kinetic energy was about $200 \mathrm{eV}$ and drift distance $92 \mathrm{~cm}$.

Very heavy ions requiring more time than allowed by the period $T$ will continue on to the detector and arrive at uncorrelated times, and may appear as broad peaks at times-of-flight not related to their mass by eq 13. As stated above, the entire vacuum system was purged with dry nitrogen to reduce the presence of such mass echoes. Clearly, uncorrelated signal due to slow moving ions in $u$-GEMS is an undesirable effect that would be difficult to handle in practice. The deflection achieved in a SDEA-based GEMS solves the problem by virtue of the deflection between the parallel plates of the SDEA.

The resulting spectrum obtained with $u$-GEMS is shown in Figure 6. It shows the time-integral of the TOF spectrum similar to that of Figure 3. The raw data shown in blue in Figure 6 show two large shoulders labeled on the right as "cumulative counts." Taking the derivative of the raw data gives the TOF spectrum, shown in red and labeled on the left as "delta counts." We note that the energy spread of the discharge ion source is typically very large, between 3 and $10 \mathrm{eV}$, and that energy spread is responsible for the widths of the observed peaks corresponding to $\mathrm{N}^{+}$and $\mathrm{N}_{2}^{+}$. The derivative of the raw data also shows two very shallow peaks corresponding possibly to water vapor $\mathrm{H}_{2} \mathrm{O}$ and to Argon gas.

The constant background observed beginning at zero drift-time and extending to the $\mathrm{N}^{+}$shoulder is due to UV photons from the Colutron discharge ion source, itself also an excellent photon source, often used for vacuum UV photon experiments and tests. This photon background illustrates a problem encountered in space applications with Hydrogen Ly- $\alpha$ and He emissions in the Earth's geocorona. This problem is greatly reduced in a GEMS designed with a well-defined SDEA cavity because the photons do not have a direct path to the detector. 


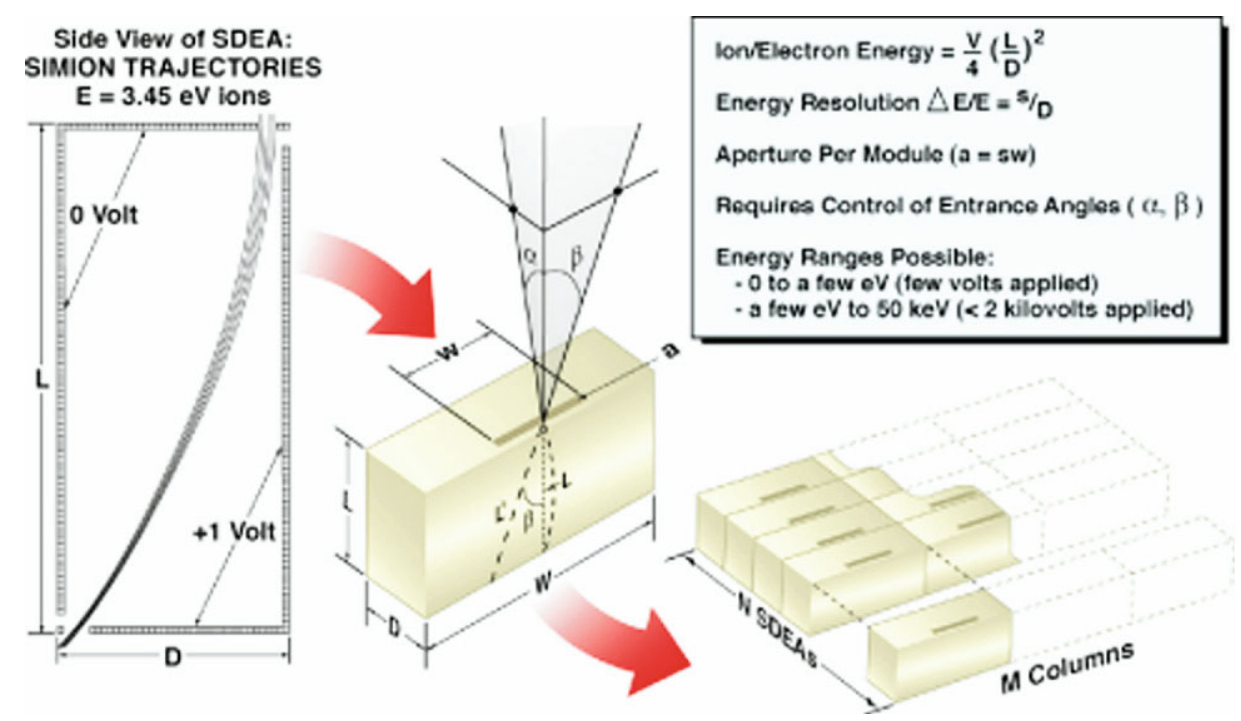

Figure 7. Summary of GEMS features and the notion that the GEMS rectangular shape enables making arrays of mass spectrometers with each GEMS looking in the same direction and analyzing the same mass range.

\section{Summary and Outlook}

True miniaturization requires re-definition, not just shrinking in size. This attitude drove the approach to GEMS to achieve a simple device that preserved the main functions of a mass spectrometer. It has attractive features as a miniature mass spectrometer with its promise of high-resolution, its compactness and simple rectangular geometry, the relatively low operating voltages, and the small number of electrodes. Its main disadvantage lies in the fact that the raw data come in the form of the time-integral of the TOF spectrum, and soon its performance must be tested to see how much of a disadvantage this feature may be.

In addition to space physics applications, GEMS may be applied in several areas of importance to monitor the atmosphere and in harsh environment applications, e.g., in analysis of gases from volcanic eruptions. Its size is determined mainly by resolving power requirements as with any other spectrometer but, with careful attention to design, as derived above, the size of the overall mass spectrometer may be kept to linear dimensions of a few $\mathrm{cm}$ for resolving powers of 1000 to 5000 .

Figure 7 summarizes the SDEA approach to GEMS to illustrate the advantages of its compactness: it illustrates SDEA operation on the left, a three-dimensional rendition of the SDEA in the middle, and the lower right panel shows how sensitivity may be enhanced in an array of small GEMS. Such an array may be deployed on a spacecraft orbiting the Moon to examine ions sputtered from the lunar surface by the solar wind with multiplied sensitivity - in this application, all the GEMS spectrometers operate in unison, only enhancing sensitivity. A collimator array mounted above the NxM array is left out to simplify the illustration. Back on the left panel, a SIMION simulation for $L / D$ ratio of 2.3, smaller than would be used in a high-resolution GEMS, illustrates the departure from parallel trajectories in a SDEA with $L / D<4$; the simulation shows a noticeable focusing or narrowing of the bundle of incident ions at the exit slit. The middle panel illustrates the angles of incidence $(\alpha, \beta)$ and the rectangular shape of the SDEA, and thereby GEMS. Detecting ambient ions in space may be accomplished in an array of $50 \times 20$ for 1000 GEMS, each with rectangular dimensions of thicknesswidth-height $=1.1 \mathrm{~cm} \times 2 \mathrm{~cm} \times 6 \mathrm{~cm}$ with a slit 0.015 $\mathrm{cm}$ wide and $0.66 \mathrm{~cm}$ long with mass resolving power $M / \Delta M=65$. The whole array of 1000 GEMS mass spectrometers would extend over an area of a little more than $55 \mathrm{~cm} \times 40 \mathrm{~cm}$ (about $22 \times 16$ in., the size of a common household furnace filter) with a likely thickness of 6 to $7 \mathrm{~cm}$. This is a large footprint for the detectors because typical MCP detectors do not exceed $10 \mathrm{~cm}^{2}$, but the detectors may be tiled atop the electronics box and treated to have sensitive areas only above each of the 1000 anodes. The size of the array aperture is selected to give total ion count rate of about $10^{5} / \mathrm{s}$.

All mass spectrometers require ion beam preparation, i.e., focusing, acceleration, and collimation. GEMS requires good collimation in the angle $\alpha$ even for modest resolutions and in the angle $\beta$ only for very high resolutions. The requirement of small slits and high degree of collimation for high-resolution work reduces the efficiency of the spectrometer if the incident ion beam has wide divergence and large energy spread. However, this was a problem that received attention decades ago, and monochromatic ion sources were developed to yield energy spreads about $0.05 \mathrm{eV}$ [11, 26]. A GEMS operating with an ion source of $0.1 \mathrm{eV}$ energy spread and with its ions accelerated to $300 \mathrm{eV}$ may require a gate pulse amplitude of only $60 \mathrm{~V}$ and achieve a resolving power of 300/0.1 $=3000$ without a monochromator in front of the GEMS. It is not difficult 


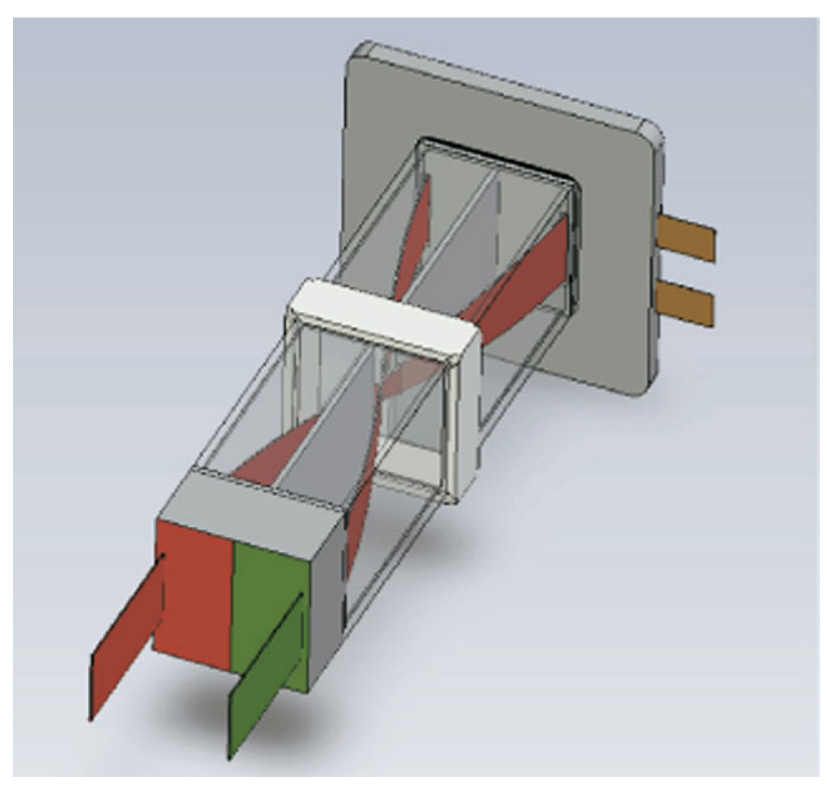

Figure 8. Dual cavity GEMS.

to see from the description of SDEA that a SDEA monochromator may be interposed between the ion source and the GEMS to further increase resolving power without increasing ion energy, with consequent reduction in gate pulse amplitude but with loss in efficiency since the monochromator would select a fraction of the ion energy distribution.

Finally, Figure 8 shows the degree of compactness that may be achieved if one takes advantage of the symmetry of the GEMS rectangle. This shows a drawing in which the deflection plates have been made transparent to reveal the internal ion motion. It shows a combination of two GEMS devices to be flown in a rocket experiment to sample both ionospheric composition (ions shown in red entering the slit on the left) and neutral air composition (neutrals shown in green entering the slit on the right) in the upper atmosphere. Incident ions (red beam on left) are accelerated to 100 $\mathrm{eV}$ kinetic energy after passing the slit on the left. Incident neutrals (green beam on right) are partially ionized inside the slit on the right and the resulting ions accelerated to $100 \mathrm{eV}$ also. Each of the two $100 \mathrm{eV}$ ion beams enters its own SDEA monochromator, shown by the two cavities with the two ion ribbons (in red) converging toward the middle of the square cavity surrounded by white walls. The two ion ribbons cross inside the white walled square cavity, changing sides, and then enter their respective GEMS analyzers where they are mass analyzed. Behind the exit slits at the back of the two GEMS are two anodes, which cannot be seen in this view of the drawing. The anode on the left detects the ions that entered as neutrals on the righthand-side slit. The anode on the right detects the ions that entered as ions on the left-hand-side slit. The height of the overall spectrometer is about $8 \mathrm{~cm}$ and provides a resolving power of 64 , sufficient to resolve atmospheric gases and metallic ions.
The GEMS compactness and simplicity of electronics lead to efficient packaging of an entire instrument requiring low pumping speeds $(<1 \mathrm{~L} / \mathrm{s})$ with relatively low voltages and telemetry to transmit data to a remote receiver.

Efficient packaging enables at least two important applications outlined below. A small GEMS can provide ground truth measurements for the NOAA GOES satellite program that supports the National Weather Service (GOES I-M Databook, prepared for NASA Goddard Space Flight Center by Space Systems/Loral.). In situ measurements of water vapor and other species like $\mathrm{CO}_{2}$ could be made from small general aviation aircraft or from unmanned aerial vehicles (UAVs) and would be used to check and cross calibrate the GOES satellite global measurements of weather and climate from geosynchronous orbit $(35,786 \mathrm{~km}$ above mean sea level). Ultimately, the development of small, low-power vacuum pumps will enable 100 to $200 \mathrm{~g}$ GEMS packages that could presumably launch on radiosondes to provide routine groundtruth measurements to support the GOES satellite measurements and other NOAA NWS programs.

Volcanic eruption studies already use mass spectrometers to sample emitted gases from fumaroles where the temperature may vary from $100^{\circ} \mathrm{C}$ to about $1000^{\circ} \mathrm{C}$ [27]. It is known that gas composition in volcanic fumaroles yield valuable information on volcanic activity and pending eruptions. There is a richness of gaseous composition in fumaroles, e.g., $\mathrm{H}_{2}, \mathrm{H}_{3}, \mathrm{HD}$, ${ }^{3} \mathrm{He},{ }^{4} \mathrm{He}, \mathrm{CH}_{4}, \mathrm{H}_{2} \mathrm{O}, \mathrm{H}_{2} \mathrm{~S}, \mathrm{~N}_{2}, \mathrm{CO}$, Ar, $\mathrm{CO}_{2}$, with many challenges to mass spectrometers; perhaps the most significant being the complete separation of ${ }^{3} \mathrm{He}$ from $\mathrm{H}_{3}$ and $\mathrm{HD}$, which is ensured by a resolving power of 600 at the 5\% peak height [28]. An implementation of GEMS with a total length of $6 \mathrm{~cm}$ (including a monochromator) is capable of 1000 resolving power to enable the separation of ${ }^{3} \mathrm{He}$ from $\mathrm{H}_{3}$ and $\mathrm{HD}$ in such applications. The compactness of GEMS and the small number of electrical leads make it possible to package such a mass spectrometer with telemetry in an enclosure that would withstand temperatures between $100{ }^{\circ} \mathrm{C}$ and $1000^{\circ} \mathrm{C}$.

This article introduced the gated electrostatic mass spectrometer (GEMS), described its basic characteristics, and showed preliminary results based on a simplified version of GEMS. The analysis obtained with SIMION and with parallel plate analyzer equations support the GEMS idea, and suggests areas of possible application and development based on the characteristics of GEMS.

\section{Acknowledgments}

The authors gratefully acknowledge the assistance of Christine Zaruba, who thought up the acronym SDEA, and Patrick Roman and Robert Kilgore, who drew up two of the figures. Ted Finne drew the 3-D picture of the double GEMS. The initial phase of this project was funded by the Director's Discretionary Fund program 
at the NASA Goddard Space Flight Center. The work leading to this paper was completed with the support of the NASA GOES-R Project Office and the NOAA GOES-R Program Office.

\section{References}

1. Nier, A. O.; Hayden, J. L. A Miniature Mattauch-Herzog Mass Spectrometer for the Investigation of Planetary Atmospheres. Int. J. Mass Spectrom. Ion Phys. 1972, 6, 339-346.

2. Nier, A. O.; Hanson, Seiff, A.; McElroy, M. B.; Spencer, N. W.; Duckett, R. J.; Knight, T. C. D.; Cook, W. S. Composition and Structure of the Martian Atmosphere: Preliminary Results from Viking I. Science 1976, 193, 786-788

3. Trinks, H.; Offermann, D.; von Zahn, U.; Steinhauer, C. J. Geophys. Res. 1978, 83, 2169-2176.

4. Schaefer, E. J.; Nichols, M. H. Mass spectrometer for upper air measurements. Am. Rocket Soc. J. 1961, 31, 1773-1776.

5. Narcisi, R. S.; Bailey, A. D. Mass Spectrometric Measurements of Positive Ions at Altitudes from 64 to 112 Kilometers. J. Geophys. Res. 1965, 70, 3687-3695.

6. Spencer, N. W.; Wharton, L. E.; Niemann, H. B.; Hedin, A. E.; Carignan, G. R. The Dynamics Explorer Wind and Temperature Spectrometer. Space Sci. Instrum. 1981, 5, 417-428.

7. Herrero, F., Nicholas, A. An Instrument Suite for the Vertical Characterization of the Ionosphere-Thermosphere System from $100 \mathrm{~km}$ to 700 km Altitude. Eos Trans. AGU 2008, 89(23), (Jt. Assemb. Suppl.) Abstract SA51A, 06.

8. Herrero, F., Nicholas, A., Vancil, B., Aalami, D., Zaruba, C., Beasley, L. The Wind and Temperature Spectrometer (WTS) in the Atmospheric Neutral Density Experiment (ANDE) Satellite. Eos Trans. AGU 2004, 85(47), (Fall Meet. Suppl.) Abstract SA24A, 05.

9. Pilinski, M., Palo, S., Herrero, F. Wind and Temperature Spectrometer, CEDAR 2008 Cubesats: Science and Systems Workshop, June 20th, 2008, Zermatt, UT.

10. Mattauch, J.; Herzog, R. Z. Phys. 1934, 89, 786.

11. Duckworth, H. E.; Barber, R. C.; Venkatasubramanian, V. S. Mass Spectroscopy, 2nd ed.; Cambridge University Press: Cambridge 1986, $31-36$.
12. Mirsaleh-Kohan, N.; Robertson, W. D.; Compton, R. N. Electron Ionization Time-of-Flight Mass Spectrometry: Historical Review and Current Applications Mass Spectrom. Rev. 2008, 27, 237-285.

13. Stephens, W. E. A Pulsed Mass Spectrometer with Time Dispersion Bull. Am. Phys. Soc 1946, 21(2), 22.

14. Cameron, A. E.; Eggers, D. F. Jr. An "Ion Velocitron." Rev. Sci. Instrum. $1948,19,605-607$.

15. Wiley, W. C.; McLaren, I. H. Time-of-Flight Mass Spectrometer with Improved Resolution. Rev. Sci. Instrum. 1955, 26, 1150-1157.

16. Guilhaus, M. Principles and Instrumentation in Time-of-Flight Mass Spectrometry. J. Mass Spectrom. 1995, 30, 1519-1532.

17. Mamyrin, B. A.; Schmikk, D. V. The Linear Mass Reflectron. Sov. Phys JETP 1979, 49, 762-764

18. Mamyrin, B. A. Laser assisted reflectron time-of-flight mass spectrometry. Int. J. Mass Spectrom. Ion Processes 1994, 131, 1-19.

19. Guilhaus, M.; Selby, D.; Milynski, V. Orthogonal Acceleration TOF-MS Mass Spectrom. Rev. 2000, 19, 65-107.

20. Dawson, J. H. J.; Guilhaus, M. Orthogonal-Acceleration Time-of-Flight Mass Spectrometer. Rapid Commun. Mass Spectrom. 1989, 3, 155-159.

21. de Hoffman, E.; Stroobant, V. Mass Spectrometry, Principles and Applications; John Wiley and Sons: Chichester, 2007; 87.

22. Moore, J. H. Jr.; Davis, C. C.; Coplan, M. A. Building Scientific Apparatus: A Practical Guide to Design and Construction; AddisonWesley Publishing: Reading, MA, 1983; pp. 307-316.

23. Sevier, K. D. Low Energy Electron Spectrometry; Wiley-Interscience: New York, 1972; pp. 18-37.

24. Ritzau, S. M.; Laprade, B. N.; Mrotek, S. R. Experimental Evaluation of Resistive Glass Ion Optics; Burle Electro-Optics: Sturbridge, MA, 2006 5-21.

25. Bevington, P. R.; Robinson, D. K. Data Reduction and Error Analysis for the Physical Sciences, 3rd ed.; McGraw-Hill: New York, 2002; 98-99.

26. Inghram, M. G., R. J. Hayden, (1954) Nucl. Sci. Ser. Natn. Res. Coun. 14 publ 311.

27. McMurtry, G. M., Hilton, D. R., Fischer, T. Development of a Mass Spectrometer-Based Instrument for Volcanic Gas Monitoring. The 6th Harsh Environment Mass Spectrometry Workshop; Cocoa Beach, FL, September 2007

28. McGuire, B.; Kilburn, C.; Murray, J. Monitoring Active Volcanoes: Strategies, Procedures, and Techniques. UCL Press: London, 1995; 315-319. 